\title{
Dermatologische Kleinwildjagd - Der Skabiesnachweis mittels Dermatoskop und Kanülenextraktion
}

\author{
Dermatological 'Game Hunt' - Detection of Scabies by Dermatoscopy and \\ Extraction via Needletip
}

Autor

Institut
C. Löser

Hautklinik, Hauttumorzentrum, Klinikum Ludwigshafen
Bibliografie

Dol http://dx.doi.org/

10.1055/s-0030-1256749

Akt Dermatol 2011; 37:

287-288 @ Georg Thieme

Verlag KG Stuttgart · New York

ISSN 0340-2541

\section{Korrespondenzadresse}

\section{Dr. med. Christoph Löser}

Leitender Oberarzt der

Hautklinik/Hauttumorzentrum

Rheinpfalz

Klinikum Ludwigshafen

Bremserstraße 79

67063 Ludwigshafen

loeserc@klilu.de

\section{Zusammenfassung \\ $\nabla$}

Nicht immer gelingt bei einer Skabies die Blickdiagnose. Eine verkannte Krätze, die fälschlicherweise als Ekzem behandelt wird, ist für den Patienten ebenso belastend wie Ekzeme nicht-ska-

„Glück entsteht oft durch Aufmerksamkeit in kleinen Dingen“

Wilhelm Busch

\section{Methode \\ $\nabla$}

Nach der Ganzkörperuntersuchung wird in befallenen Arealen gezielt nach Papeln oder nach Gängen gesucht, die Eier und Skybala (Milbenkot) enthalten können. Neben den typischen Prädilektionsstellen (Intertrigines, Nabelregion, Areolae, Genitalregion) werden die Fingerzwischenräume wegen des häufigen Befalls und der leichten Zugänglichkeit besonders beachtet. Die Läsionen werden dann mit einem Dermatoskop inspiziert ( $\triangle$ Abb. 1). Die Skabiesmilbe ist meist mit etwas Abstand zur Papel oder zum ekzematisierten Gang aufzufinden ( $A$ Abb. 2, 3). Sie zeichnet sich durch ein typisches, dreieckiges, dunkles Areal ab, welches der Kopfregion der Milbe entspricht und bei zehnfacher Vergrößerung an eine flache Pyramide oder eine Baskenmütze erinnert ( $\bullet$ Abb.4). Mittels einer Kanüle wird die Hornschicht über der als Milbe verdächtigten Struktur eingeritzt und vorsichtig abgehoben, dann die Milbe auf die Kanülenspitze aufgeladen und extrahiert. Zum definitiven Nachweis, insbesondere zur Unterscheidung von hämorrhagischen Krüstchen, wird das Material auf einen Objektträger gebracht und mit $\mathrm{NaCl}$ oder $\mathrm{KOH}$ unter einem Deckgläschen eingedeckt. Mit dem Mikroskop gelingt dann die Bestätigung des Jagderfolges ( $\bullet$ Abb.5). biöser Ursache, die sogenannten Sicherheitsbehandlungen mit Antiskabiosa unterzogen werden. In jedem Fall ist die Diagnosesicherung durch einen optischen Skabiesnachweis der unschlagbare Beweis und bedeutet das Ende jeder diagnostischen Unsicherheit.

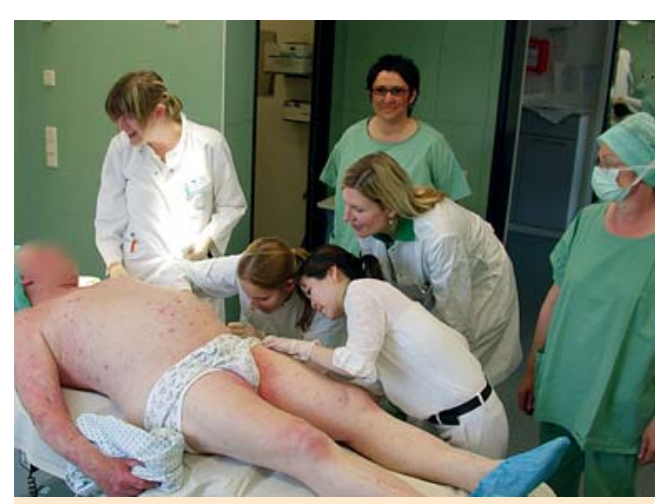

Abb. 1 Das Team (historische Aufnahme in der Hautklinik Ludwigshafen aus dem Jahr 2007; Dres. Groß, Hassel, Ha, Coordes, Frau Kunz und Frau Werner) ist vom Jagdfieber erfasst und die erfolgreiche Kleinwildjagd bewahrt den Patienten vor einer verzichtbaren Probebiopsie.

\section{Diskussion \\ $\nabla$}

Mögliche Verfahren zum Skabiesnachweis sind der Klebestreifennachweis, die Shavebiopsie, die Probebiopsie, der Gangnachweis mittels sogenanntem Burrow-Ink-Test oder die Epilumineszenzmikroskopie [1-4]. Alle diese Methoden sind nach unserer Erfahrung vergleichsweise aufwendig oder unsicher. Der oben beschriebene und seit Renucci 1835 [5] geübte Nachweis der Skabiesmilbe mittels Nadelextraktion ist durch die Anwendung eines Dermatoskopes heute stark erleichtert. Wir verwenden ein Dermatoskop 


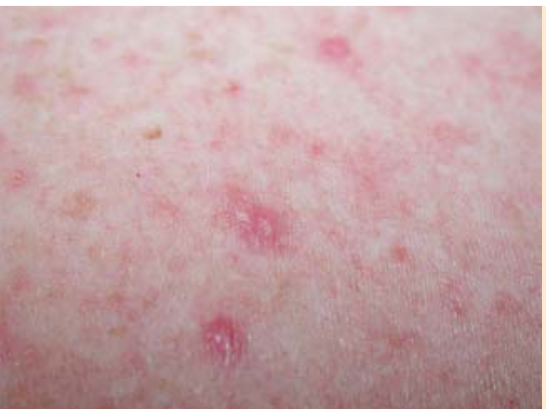

Abb. 2 Eine verdächtige Papel wurde lokalisiert.

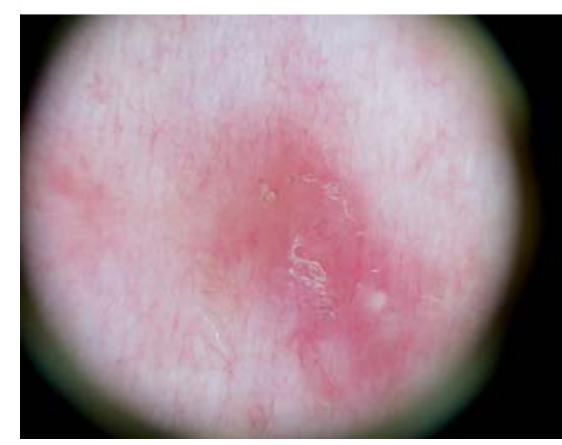

Abb. 3 Die Milbe wird mittels Dermatoskop in 10-facher Vergrößerung dargestellt, es zeigen sich auch Gangstrukturen.

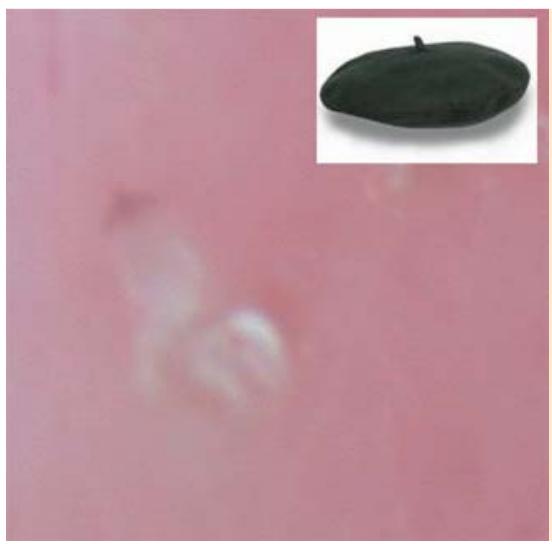

Abb. 4 Charakteristisch ist die helle, ovaläre Struktur mit einer aufsitzenden, dreiecksförmigen, dunkleren Kappe (BaskenmützenZeichen), die den vier Vorderbeinen und dem Kopf entspricht.

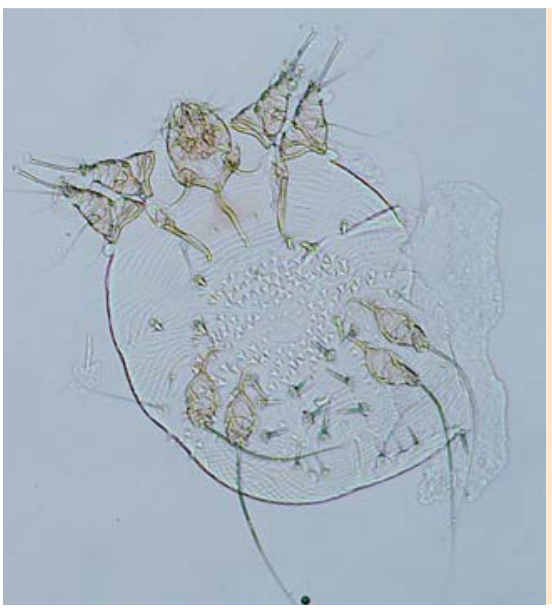

Abb. 5 Nach Extraktion mittels Kanüle unter Sicht zeigt sich mikroskopisch die wahre Schönheit der winzigen Beute.
DL100 der Marke Dermlite, welches die Milbenextraktion unter Sicht erlaubt [6].

\section{Fazit}

$\nabla$

Wenn man weiß, wo und wonach man suchen muss, ist der beschriebene Nachweis einfach und führt regelmäßig zum Erfolg.

\section{Bonus}

Im Jahr 2010 wurde die Extraktion einer Skabiesmilbe an einem typischen Ambulanztag vorgenommen und ein Deckgläschen war nicht sofort zur Hand. Die Inspektion zeigte dann unter dem Mikroskop eine lebende Milbe. Deren Wanderung kann bei Interesse im Internet unter Youtube (dance of the acarus; www.youtube.com/watch?v=4f2m5FVKXns) verfolgt werden.

\section{Abstract}

\section{Dermatological 'Game Hunt' - Detection of Scabies by Dermatoscopy and Extraction via Needletip}

Not always scabies can be diagnosed by clinical sight. When scabies is not recognized as such and treated as eczema, the patient will suffer - when treated for non-existing scabies even so. In any case the extraction of the scabies-mite is the ultimate proof and puts an end to all diagnostic uncertainty.

\section{Literatur}

1 Chouela E, Abeldan oA, Pellerano G, Hernández MI. Diagnosis and Treatment of Scabies - A Practical Guide. Am J Clin Dematol 2002; 3: 9-18

2 Katsumata K, Katsumata K. Simple Method of Detecting Sarcoptes Scabiei Var Hominis Mites among Bedridden Elderly Patients Suffering from Severe Scabies Infestation Using an Adhesive-Tape. The Japanese Society of Internal Medicine 2006; 45: 857 - 859

3 Hafner C. Skabies. Hautarzt 2009; 60: 145-159

4 Sunderkotter C, Mayser P, Folster-Holst R et al. Skabies. J Dtsch Dermatol Ges 2007; 5: 424-430
5 Crissey JT, Parish LC. The dermatology and syphilology of the nineteenth century. New York: Praeger; 1981

6 Groß M, Löser C. Kleinwildjagd: Der Skabiesnachweis. Posterpräsentation. Südwestdeutsche Dermatologische Gesellschaft. Stuttgart: 2007 